\title{
Inkjet Printing Resolution Study for Multi-Material Rapid Prototyping
}

\author{
Mustaffa IBRAHIM ${ }^{1}$, Takayuki OTSUBO ${ }^{2}$, Hiroyuki NARAHARA ${ }^{3}$, Hiroshi KORESAWA ${ }^{4}$ and \\ Hiroshi SUZUKI ${ }^{5}$ \\ 1 Department of Mechanical Information Science and Technology \\ Kyushu Institute of Technology \\ 680-4 Kawazu, lizuka Shi, Fukuoka Ken, 820-8502 Japan \\ Phone: +81-0948-29-7786, Fax: +81-0948-29-7751 \\ E-mail: c792002m@iizuka.isc.kyutech.ac.jp \\ ${ }^{2}$ E-mail: a234016t@iizuka.isc.kyutech.ac.jp \\ ${ }^{3}$ E-mail: nara@mse.kyutech.ac.jp \\ ${ }^{4}$ E-mail: koresawa@mse.kyutech.ac.jp \\ ${ }^{5}$ E-mail: suzuki@mse.kyutech.ac.jp
}

Summary

In this study a commercial inkjet printer was modified to study the resolution of fluid dot placement required to fabricate $3 \mathrm{D}$ multi-material patterns layer by layer. A JAVA based computer program was developed to convert stereolithography (STL) data layer by layer, control the ink cartridges individually and print ink with customized fluid dot placement arrangements. The study found out that complement printing between nozzles which is $30 \mu \mathrm{m}$ in diameter and $144 \mu \mathrm{m}$ apart is essential to achieve a fully dense $3 \mathrm{D}$ pattern. When printed with $36 \mu \mathrm{m}$ printing spacing vertically, a layer thickness of $1.30 \mu \mathrm{m}$ is achievable and when printing layer by layer, the thickness increases almost at a linear rate.

Keywords: Rapid prototyping, Inkjet printing, Multi-Material

\section{Introduction}

Recently a rapid prototyping method for building parts layer-by-layer has led to interest in fabrication of electrical circuits and optical devices by inkjet printing. The classic definition of rapid prototyping is "a special class of machine technology that quickly produces models and prototype parts from 3-D data using an additive approach to form the physical models" (1). The nature of the inkjet printing process prints dots, but for printing multi-material 3-D functional parts, lines and areas with good dot to dot conductivity is a necessity such as for electrical and thermal conduction. An ink-jet printer patterns material by ejecting tiny droplets of liquid ink from its print head nozzles or orifices as it moved in two dimensions approximately 1 $\mathrm{mm}$ above a substrate. The advantages of inkjet technology are straightforward: its apparent simplicity, data driven, high material deposition speed, reduction on the waste of precious materials and also eliminates cross-contamination on surfaces because it's a non-contact process. The overall size of the particles that can be jetted are limited by the nozzle exit orifice diameter and line width/ thickness is determined by the jetted liquid properties and the orifice diameter ${ }^{(2)}$. Although there are already some ongoing researches using inkjet printing to build MEMS, organic displays, and biological arrays ${ }^{(3)(4)}$, most of them are using drop on demand platform that has a single nozzle piezoelectric actuated device such as from MicroFab Technologies Inc. to direct write materials as denoted in Fig.1. In our previous study, silver nanoparticle ink which contains silver, plated gold tetradecane as solvent and have particle size between $3-7 \mathrm{~nm}$ was successfully printed using the inkjet printing technology to directly write electronic circuit ${ }^{(5)}$. This study intends to utilize JAVA program to manipulate CAD design data and interface them with a modified commercial piezoelectric printer that has 357 nozzles to produce 3-D patterns as shown in Fig. 2. The use of multiple nozzles in printing 3-D structures will inevitably increase fabrication speed when a larger area is anticipated. The printer was also equipped with motorized stages and a heater in order to fabricate $3 \mathrm{D}$ patterns.

The focus of the experiments was to study the deposited ink covering area horizontally and vertically in order to print 3D patterns. Regions that were not deposited by the fluid will give a significant effect if functional ink was to be used.

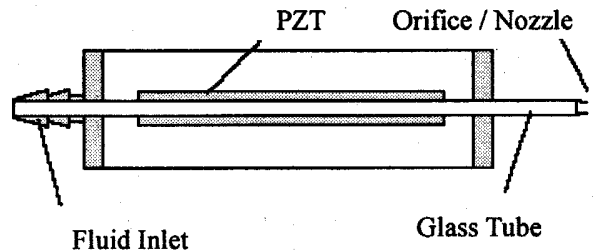

Fig.1 Single channel drop-on-demand dispensing device configuration (www.microfab.com)

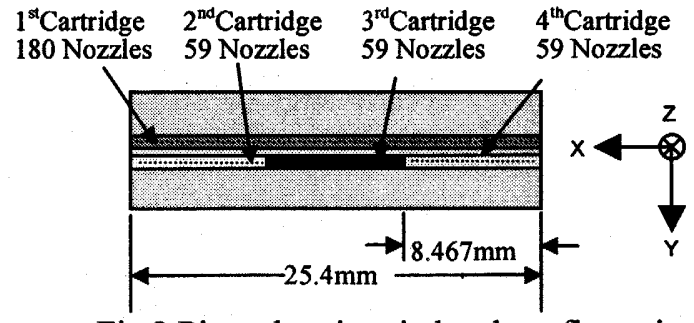

Fig.2 Piezoelectric printhead configuration 


\section{Materials and Methods}

Although there are numerous types of inkjet technology, the most common is the drop on demand inkjet systems which have dominate the lower-end printer market known as the thermal and the piezoelectric technology. In thermal inkjet technology, the drop is initiated by heating the ink to create a bubble until the pressure forces it to burst and hit the paper. However the high heat temperatures may trigger problems or incompatibility with certain functional inks formulations. The other inkjet technology which was used in this study is from Epson, utilizes a piezoelectric crystal positioned at the rear of the inkjet reservoir. When actuated, the piezoelectric crystal flexes and forces a drop of ink out of the nozzle ${ }^{(6)}$.

This study is using Java program to slice rapid prototyping STL data and translate them to bitmap data layer by layer. The layer image was then sent to the printer which was also control by the Java program so that it was feasible to choose the inkjet cartridge individually for color selection and to avoid the color mixing process as for the normal printer would operate. The inkjet printing system horizontal movement is not modified but the mechanical paper feed mechanism was replaced by the motorized stage as depicted in Fig.3. The java program synchronizes the motorized stages movement with the printer in order to fabricate 3D patterns identical to the Rapid prototyping concept. A film heater was also placed on top of the stage to dry the deposited fluids and to maintain their shape rather than spreading out on the surface and causing dots to interact with one another. The printer print head nozzle is about $30 \mu \mathrm{m}$ in diameter distance in-between nozzles are $144 \mu \mathrm{m}$.

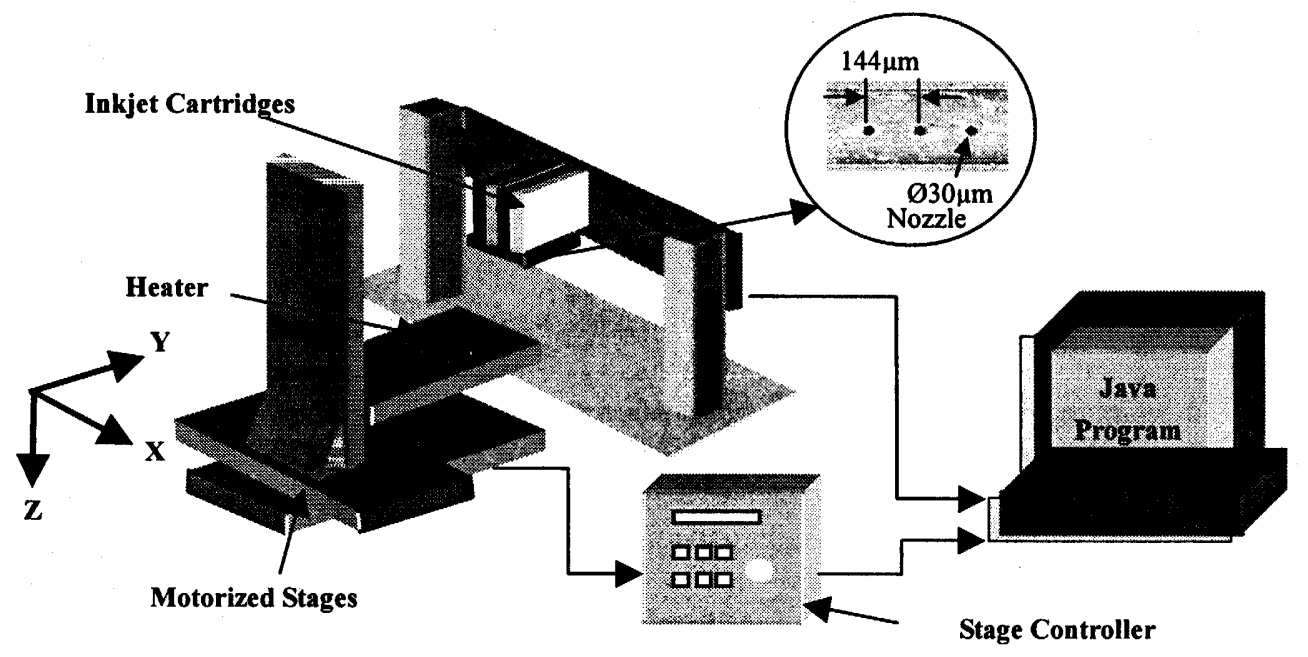

Fig.3 Inkjet printing system

Firstly, the ink drying temperature must be determined because partial drying will cause the ink to appear dry on the surface while trapping solvent underneath the surface. A film heater was used and the voltage supplying the film heater was varied to find the required time and temperature to dry the ink. To makes sure the heat was evenly distributed, a copper plate was placed on top of the heater. A tape pull test was than done to make sure that the ink adheres well to the substrate. The spreading of the ink deposited was then studied by printing the ink on different substrate material which is stainless steel plate, glass and inkjet film. Although we know that the nozzle size is about $30 \mu \mathrm{m}$, the actual size of printed dot must be determined. The spreading of the ink is important if one is trying to get a precise deposition pattern in inkjet printing. This experiment was done to study the ink spreading area on different materials when the same volume of ink was deposited so that spacing for complement printing distance can be established. The printed line width and its average roughness $(\mathrm{Ra})$ was measured and recorded.

Resolution, the basic term used to classify printers and is most commonly expressed by the unit dots per inch (dpi) denotes the number of clearly resolved points a printhead theoretically can print in a one-inch interval. The horizontal resolution is defined by the firing frequency of the printhead and the linear speed of its horizontal movement while the vertical resolution of a printer depends on the positioning accuracy of the mechanical paper feed. In this study however, since the mechanical paper feed was removed and replaced by the motorized stage, ink positioning accuracy on vertical axis was determined by complement printing or overlaying the ink between the two adjacent nozzles which is $144 \mu \mathrm{m}$ apart on slide glass. Surface roughness parameters $\mathrm{Ra}$ - parameter for measuring the average roughness of a surface, was measured from 1 complement printing up to 11 complement printing. The ability of inkjet printing technology in arranging dots precisely to create layers of material makes it interesting in layering multiple materials that is integrated in one part. By printing layer by layer on top of one and another layer thickness of 1,3,5,7 and 9 layers was 
measured and recorded. In this study, experiment was also done on fabricating part that consists of mechanical structure and electrical elements such as printing a schematic of a capacitor pattern on a surface of different heights using pigment ink for positional verification as described in Fig.4.

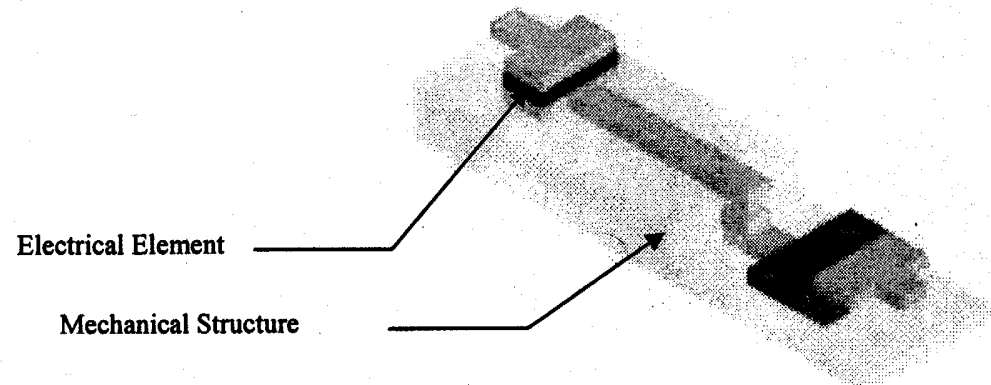

Fig.4 Integration of mechanical and electrical element in a part

\section{Experimental Results}

From this simple experiment, the results shows that increasing drying temperature will reduce the drying time and at $80^{\circ} \mathrm{C}$ ink will dry for about 10 seconds as depicted in Fig.5. Although we can use higher temperature to shorten the drying time, care must also be taken on the effect of the heat on the nozzles surface to avoid the ink from drying and clogging the printhead nozzles. The heat should be just enough to evaporate the solvents in the ink. The results shown in Fig. 6 indicate that ink printed on three different positions with two different materials which is stainless steel plate, glass and inkjet film gave different spreading area. The width of the line printed on glass is less compared with ink printed on stainless plate and inkjet film. This shows that the ink spread less on glass because its contact angle which determines its wetting is bigger compared to ink printed on the other substrate. The average roughness value of ink printed on glass is also less than the other materials.

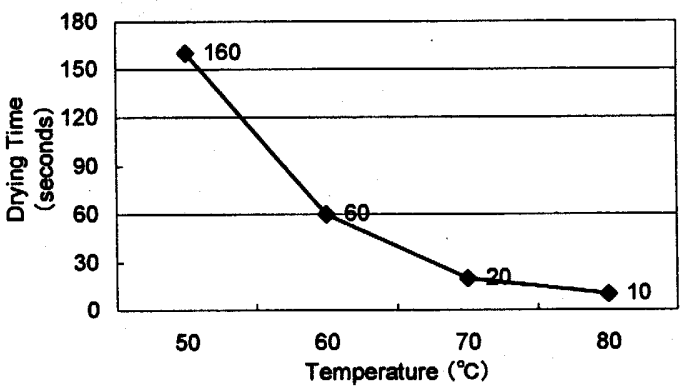

Fig.5 Drying Time

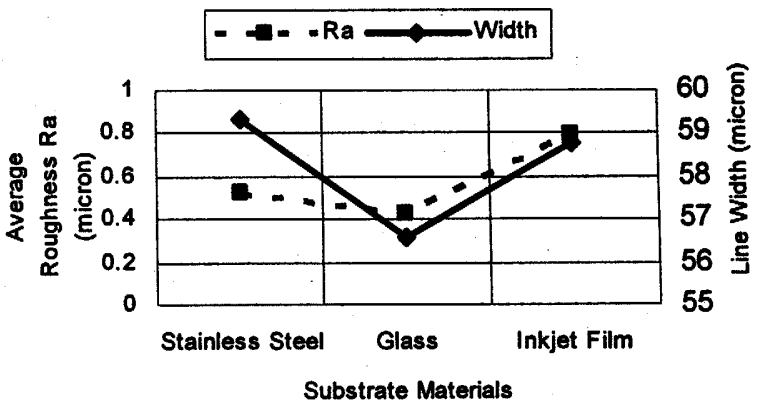

Fig.6 Ink Spreading

Experiments done on complement printing to get a fully dense pattern. Fig.7a shows the result of printing without complement where there was gap of about $90 \mu \mathrm{m}$ in-between the two adjacent nozzles. When one complement printing was done, the gap is still not fully covered by the ink because the width of a dot is only about $55 \mu \mathrm{m}$ as shown in Fig. $7 \mathrm{~b}$. In order to fully fill in the gap between the two adjacent nozzles a minimum of 2 complement printing which is $48 \mu \mathrm{m}$ apart is necessary as shown in Fig.7c. With nozzle size of $30 \mu \mathrm{m}$ in diameter, $144 \mu \mathrm{m}$ apart and printed at a horizontal resolution of $360 \mathrm{dpi}$, the smallest dot can be printed is about $55 \mu \mathrm{m}$ horizontally and vertically is about $150 \mu \mathrm{m}$ which is from 1 pixel square image as denoted in Fig.8. Overlay printing until 11 complement in between the two nozzles shows that the roughness of the surface is increasing as more lines were printed with smaller printing spacing as shown in Fig. 9

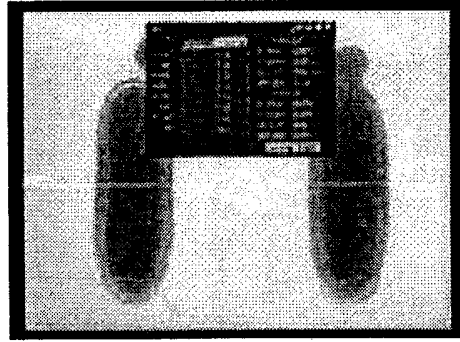

Fig. 7a Without complements

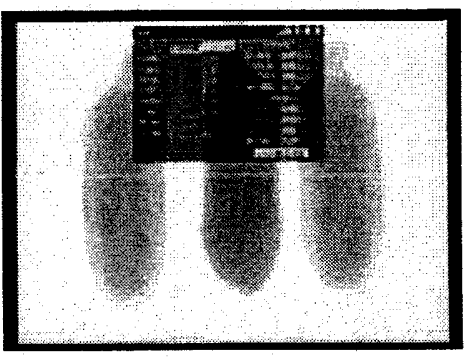

Fig.7b One complement

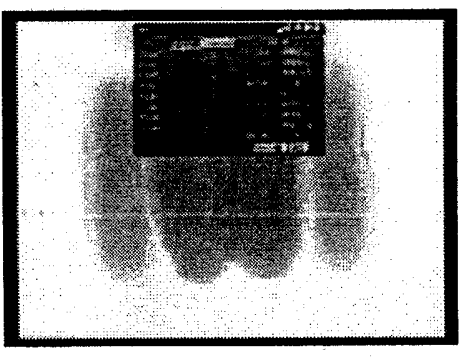

Fig.7c Two complements 


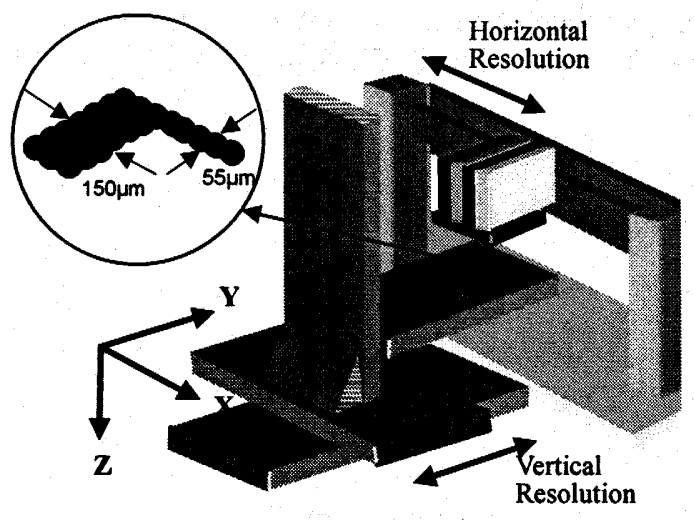

Fig.8 Printing resolution

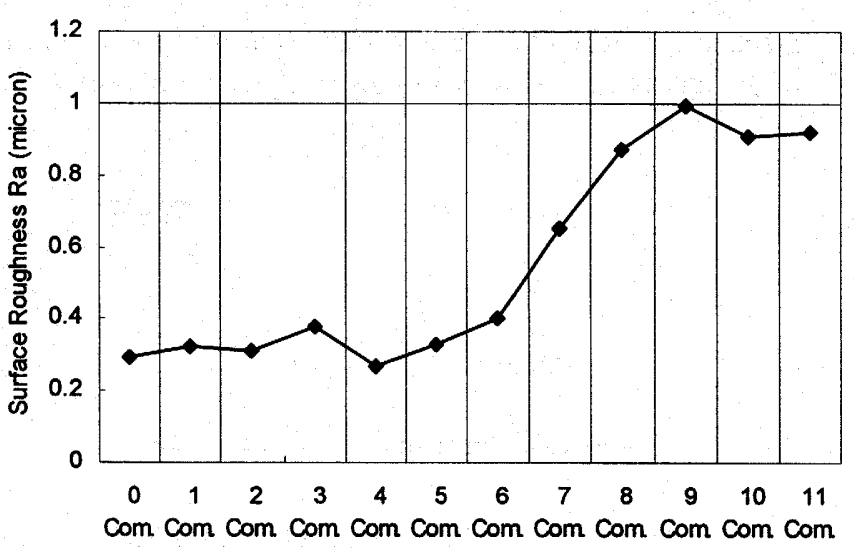

Fig.9 Complement Printing Surface Roughness

For the layer thickness experiment, a $36 \mu \mathrm{m}$ printing spacing was used to print square pattern and thickness differences was measured. The results indicate that when the first layer was printed on the slide glass the edge of the ink tend to spread on the glass surface. As the number of layers is increased there is almost a linear increase in the average thickness of the pattern as depicted in Fig.10. For the experiment on printing a capacitor pattern depicted in Fig.11, two types of ink was used which are cyan representing conductive ink for the electrode and magenta representing dielectric ink. The bottom electrode, dielectric and the top electrode layers are laid down successively. Both the area and the thickness of the dielectric could be varied to select the value of the capacitance.

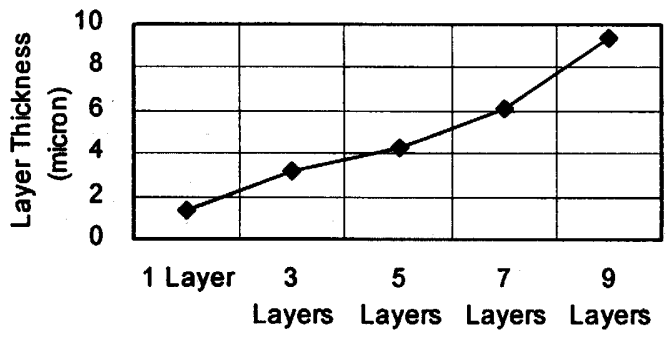

Fig.10 Layer Thickness

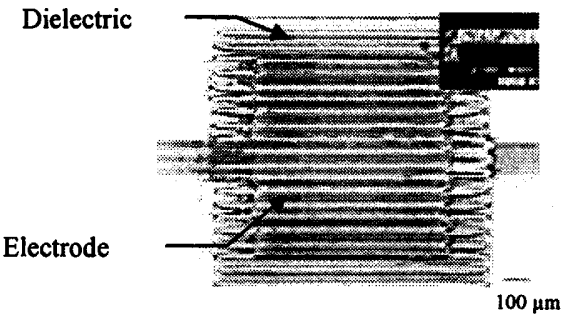

Fig.11 Inkjet printing of capacitor model

The capacitor was printed on the surface of the mechanical structure represented by the yellow ink. Structure with different surface height was fabricated by printing different number of layers where 1 layer is roughly about $1 \mu \mathrm{m}$ thick. The images described in Fig.12 shows the use of inkjet printing to fabricate multiple material 3D patterns with reference to the Rapid Prototyping technology which fabricate 3D object layer by layer.

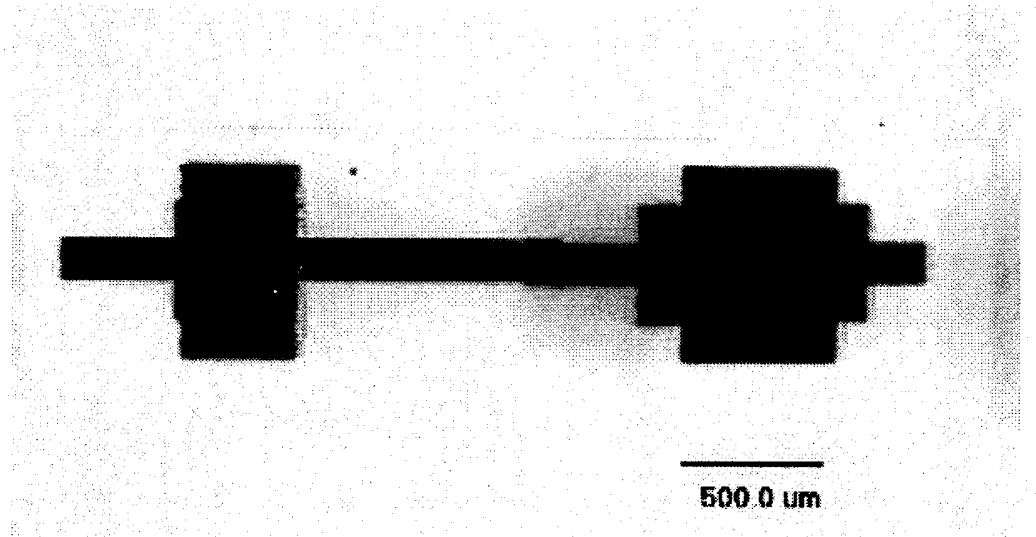

Fig.12b Inkjet printing of part with capacitor model 


\section{Discussion}

The ability to converts CAD design data and control the inkjet printing machine within Java program gives us the flexibility to harmonize different equipment in the same environment. Commercial inkjet printer was modified and transform into a machine that can fabricate 3D structure.. Although there are still many improvement has to be made in terms of hardware and software, the capability of the inkjet printing technology in depositing material layer by layer to build 3D structure is promising. Some parameters of the machine such as firing frequency still needed to be control if fluid with different viscosity and surface tension needed to be deposited. The nozzles must also be taken care off because heat interaction on fluid can clog the opening of the nozzles. If particles are clogging parts of the orifice or nozzle both horizontal and vertical resolution can be affected and a deviation of 1 degree can cause dot misplacement of a few microns. This will also cause 'satellite drops' which are unwanted drops of ink that take off from the main droplets.

\section{Conclusion}

From our previous research of inkjet printing of silver ink, it's quite difficult to get a flat pattern due to the nature of the inkjet printing process and the design of the printer head nozzles. The fluid property requirements for demand-mode inkjet dispensing are much influence by its viscosity and surface tension range. The study on inkjet printing resolution using pigment ink was necessary prior to printing functional inks because fluids such as metal filled conductive inks or polymer inks are not cheap and extra care must be taken in handling these materials. The inkjet printing machine that we build shows the flexibility in depositing multiple material fluid and number of complement printing needed to build 3D structures layer by layer using Java program. The program can also be use to generate one ink cartridge (tool) path file for every different material needed to be deposited using the inkjet printing technology. From the experiments done to explore the ink deposition resolution using a commercial piezoelectric printer a few conclusions can be made:

- When printing at a horizontal resolution of $360 \mathrm{dpi}$, the minimum width achievable is $55 \mu \mathrm{m}$ in the $\mathrm{X}$ axis and about $150 \mu \mathrm{m}$ in the $\mathrm{Y}$ axis.

- The minimum complement printing distance necessary to connect dots in the $Y$ axis is about $48 \mu \mathrm{m}$ which is 2 complement printing in-between nozzles which are $144 \mu \mathrm{m}$ apart.

\section{References}

(1)T. Grimm and T. Wohlers, Time Compression Technologies, May 2001

(2)Wallace, D.B, Cox, W.R and Hayes, D.J, "Direct Write for Ink-Jet Techniques", in Direct Write Technologies for Rapid Prototyping Applications- Sensors, Electronics, and Integrated Power Sources, eds., A. Pique and D.B. Chrisey, Academic press, New York, 2002.

(3)Hayes DJ, Cox WR, Wallace DB, "Printing System for MEMS Packaging", Proceedings, SPIE Micromachining \& Microfabrication Conference, San Francisco,CA, October 22-25, 2001.

(4)Shah V.G, Hayes D.J, "Trimming and Printing of Embedded Resistors Using Demand-Mode Ink-Jet Technology and Conductive Polymer. Proc., IPC Printed Circuits,Expo 2002, Long Beach, CA, March 24-28, 2002.

(5)Mustaffa B.I., H. Narahara, H. Koresawa and H. Suzuki,"Integration Of Silver Nanoparticle Inkjet Printing And Layered Manufacturing Technology", The $7^{\text {th }}$.Intl. Conf. Quality in Research (QIR) 2004 (Mechanical Engineering), ME-DM-04-1-4, Jakarta, Indonesia, August 2004.

(6)R. Andrew McGill, Bradley Ringeisen, Peter K. Wu, "The Role of Direct Writing For Chemical and Biological Materials: Commercial \& Military Sensing Applications", in Direct Write Technologies for Rapid Prototyping Applications- Sensors, Electronics, and Integrated Power Sources, eds., A. Pique and D.B. Chrisey, Academic press, New York, 2002. 